\title{
Magnetic responsive hydroxyapatite composite scaffolds construction for bone defect reparation
}

\author{
This article was published in the following Dove Press journal: \\ International Journal of Nanomedicine \\ 3 July 2012 \\ Number of times this article has been viewed
}

\section{Xiao Bo Zeng \\ $\mathrm{Hao} \mathrm{Hu}$ \\ Li Qin Xie \\ Fang Lan \\ Wen Jiang \\ Yao Wu \\ Zhong Wei Gu}

National Engineering Research Center for Biomaterials, Sichuan University, Chengdu, Sichuan, People's Republic of China
Correspondence: Zhongwei Gu National Engineering Research Center for Biomaterials, Sichuan University, Chengdu 610064,

People's Republic of China

Tel +8628854l 0336

Fax +86 2885410653

Email zwgu@scu.edu.cn

Yao Wu

National Engineering Research Center for Biomaterials, Sichuan University,

Chengdu 610064,

People's Republic of China

Tel +86 288 54| 4308

Fax +86 2885410653

Email yaowu_amanda@I26.com
Introduction: In recent years, interest in magnetic biomimetic scaffolds for tissue engineering has increased considerably. A type of magnetic scaffold composed of magnetic nanoparticles (MNPs) and hydroxyapatite (HA) for bone repair has been developed by our research group.

Aim and methods: In this study, to investigate the influence of the MNP content (in the scaffolds) on the cell behaviors and the interactions between the magnetic scaffold and the exterior magnetic field, a series of MNP-HA magnetic scaffolds with different MNP contents (from $0.2 \%$ to $2 \%$ ) were fabricated by immersing HA scaffold into MNP colloid. ROS 17/2.8 and MC3T3-E1 cells were cultured on the scaffolds in vitro, with and without an exterior magnetic field, respectively. The cell adhesion, proliferation and differentiation were evaluated via scanning electron microscopy; confocal laser scanning microscopy; and 3-(4,5-Dimethylthiazol-2-yl)-2,5diphenyltetrazolium bromide (MTT), alkaline phosphatase, and bone gla protein activity tests. Results: The results demonstrated the positive influence of the magnetic scaffolds on cell adhesion, proliferation, and differentiation. Further, a higher amount of MNPs on the magnetic scaffolds led to more significant stimulation.

Conclusion: The magnetic scaffold can respond to the exterior magnetic field and engender some synergistic effect to intensify the stimulating effect of a magnetic field to the proliferation and differentiation of cells.

Keywords: magnetic therapy, magnetic nanoparticles, bone repair, magnetic responsive

\section{Introduction}

Magnetic therapy has been considered a promising alternative in disease treatments in health care, especially in the treatments of bone diseases. Research has indicated that magnetic fields may stimulate the proliferation and differentiation of osteoblasts, promote the expression of growth factors such as bone morphological protein, increase osteointegration, and accelerate new bone formation. ${ }^{1-4}$ Magnetic fields were also found to be beneficial in promoting the integration of bone and implants, increasing bone density and calcium content, and accelerating the healing of bone fractures..$^{5-8}$ Among the magnetic materials usually used in the biomedical field, magnetic nanoparticles (MNPs) have drawn great interest owing to their unique magnetic properties, including the fact that they become superparamagnetic at diameters of $<20 \mathrm{~nm} .{ }^{9}$ Coupled with their excellent biocompatibility, MNPs have been widely used in biomedical applications such as drug delivery, magnetic resonance imaging reagents, bio-separation, enzyme immobilization, and magnetic hyperthermia. ${ }^{10-13}$ But their application in tissue regeneration, especially in bone repair, has seldom been considered. 
To develop a novel bone-tissue engineering scaffold, a few research groups have tried to merge superparamagnetic nanoparticles into some bone substitutes to produce magnetic scaffolds. To the best of our knowledge, very few studies have involved magnetic scaffolds for bone regeneration. ${ }^{1421}$ Wei et al developed magnetic biodegradable fibrous materials with potential in bone regeneration, which exhibited good cell adhesion and proliferation. ${ }^{14}$ Bock et al fabricated a kind of magnetic scaffold by loading MNPs into the conventional hydroxyapatite (HA)/collagen scaffold. ${ }^{21}$ The in vitro study demonstrated that this kind of magnetic scaffold had no negative influence on human bronchial smooth muscle cells. ${ }^{21}$ However, little research has focused on the combined synthetic effects of magnetic scaffolds and exterior magnetic fields or the influence of magnetic scaffolds' MNP content on cell behaviors.

As mentioned, at a size of $<20 \mathrm{~nm}$, the MNPs become nonmagnetic on a macroscopic scale and behave like common materials in the absence of an exterior magnetic field. However, each particle could be considered a single magnetic domain, providing a magnetic field at a nanoscale, which makes it possible for magnetic scaffolds to provide an intrinsic magnetic therapy. Meanwhile, once exposed under an exterior magnetic field, MNPs will be rapidly magnetized to saturation and respond to the exterior magnetic field to provide enhanced magnetic therapy. ${ }^{22-24}$ Therefore, MNPs will play a very important role in the functionality of magnetic scaffolds and cell behaviors are likely to be influenced by the content of MNPs in the magnetic scaffolds. It is also important to investigate the response of magnetic scaffolds to the exterior magnetic field so as to determine if enhanced magnetic therapy could be achieved.

Accordingly, our research group devised a new strategy to enhance bone tissue regeneration: fabricating a new type of magnetic scaffold via the integration of superparamagnetic nanoparticles into calcium phosphate bioceramics. In this new type of magnetic scaffold, MNPs are firmly integrated into calcium phosphate bioceramics through capillary force. In this way, an excellent level of magnetic therapy was successfully integrated into the traditional bone graft substitute. Our preliminary study indicated that this type of magnetic scaffold could significantly promote the proliferation and differentiation of the osteoblast cells. ${ }^{25}$ However, the influence of MNP content and the response of the magnetic scaffold to the exterior magnetic field have not been investigated systematically. Therefore, in this study, MNP-HA (MHA) magnetic scaffolds with different MNP contents were prepared by immersing HA into MNP colloid. Osteoblast and preosteoblast cells were cultured on the scaffolds with and without exterior electromagnetic fields, respectively, to explore the influence of MNP content on bone formation and growth as well as to investigate the cellular responses to the magnetic scaffolds and the exterior magnetic field in vitro.

\section{Materials and methods Preparation of the MHA and HA scaffolds}

Synthesis of MNPs

MNPs were synthesized following the method reported by Sun and Zeng. ${ }^{26}$ Briefly, 2 mmol of iron (III) acetylacetonate was suspended in $20 \mathrm{~mL}$ of phenyl ether together with $10 \mathrm{mmol}$ of 1,2-hexadecanediol, $6 \mathrm{mmol}$ of oleylamine, and $6 \mathrm{mmol}$ of oleic acid. The mixture was first heated to $200^{\circ} \mathrm{C}$ for 2 hours and then heated to reflux for 1 hour in an argon atmosphere before being allowed to cool to room temperature. Then the suspension was precipitated with ethanol and the sediment was isolated from the solvent by magnetic decantation. The precipitates were dispersed in n-hexane and centrifuged for 10 minutes at $8000 \mathrm{rpm}$ to remove all undispersed residues. Following this, the black n-hexane dispersion was precipitated with ethanol again and the solvent was removed through magnetic separation. Finally, MNPs were re-dispersed in n-hexane to form stable colloids with different concentrations.

\section{Preparation of the HA scaffolds}

In this study, $50 \mathrm{~g}$ of HA powder (provided by the National Engineering Research Center for Biomaterials, Sichuan University, Chengdu, China) was mixed with $25 \mathrm{~mL} \mathrm{5 \%}$ polyethylene glycol 6000, $20 \mathrm{~mL} 1 \%$ methyl cellulose, $45 \mathrm{~mL}$ $\mathrm{H}_{2} \mathrm{O}$, and $15 \mathrm{~mL} 30 \% \mathrm{H}_{2} \mathrm{O}_{2}$ in a container and dispersed under ultrasonic vibration for 20 minutes. The slurry was then foamed by microwave-assisted heating, shaped into columns, and dried at $80^{\circ} \mathrm{C}$ for 24 hours. The green bodies obtained were sintered at $1100^{\circ} \mathrm{C}$ for 2 hours to obtain the ceramics. Finally, the ceramics were cut into disks of $12 \mathrm{~mm}$ diameter and $2 \mathrm{~mm}$ height.

\section{Preparation of the MHA scaffolds}

The HA disks were immersed in the MNP colloid to allow infiltration of the MNPs into the pores of the scaffolds by capillary force. The disks were then taken out to volatilize the hexane completely. By tuning the duration of immersion and the concentration of the MNP colloid, a series of MHA 
scaffolds with different MNP contents $(0.2,0.4,0.5,1.0,1.5$, $2.0 \mathrm{wt} \%$ ) was prepared, as presented in Table 1.

\section{Characterization of the magnetic scaffolds}

Morphological evaluation of the MHA and HA scaffolds was performed using a scanning electron microscope (JEM-100CX; JEOL, Tokyo, Japan) equipped with an energydispersive spectrometer link microprobe.

The MNP content of the MHA scaffolds was characterized by precision balance (XP205; METTLER TOLEDO, Chicago, IL) and atomic absorption spectroscopy (AAnalyst 800 Spectrometer; PerkinElmer, Waltham, MA).

Powder X-ray diffraction (XRD) (X'Pert Pro MPD; PANalytical, Almelo, The Netherlands) was employed to study the crystal structures of the MHA and HA scaffolds, with angles ranging from $10^{\circ}$ to $80^{\circ}$.

Magnetic properties were evaluated using a vibrating sample magnetometer (Model BHV-525; Riken Denshi Co, Ltd, Tokyo, Japan) with field from 0 to 15,000 Oe at $300 \mathrm{~K}$.

\section{Stability of MHA scaffolds under biological solutions}

The release behaviors of MHA1 were assessed in vitro in four kinds of media: Milli-Q water, phosphate-buffered saline (PBS), simulated body fluids (SBF) and fetal bovine serum (FBS). The specimen was immersed into $100 \mathrm{~mL}$ of fluid and shaken ceaselessly at $37^{\circ} \mathrm{C}$. A $2 \mathrm{~mL}$ aliquot of leaching solution was taken away at each time point (2, 4, 8, and 12 hours, and 1, 2, 3, and 5 days), while the same volume of fresh medium was simultaneously added back into the solution. The samples were further diluted eightfold for atomic absorption spectroscopy analysis to evaluate the iron content and calculate the masses of released magnetite.

Table I Preparation parameters for all types of magnetic scaffolds

\begin{tabular}{llll}
\hline $\begin{array}{l}\text { Type } \\
\text { of MHA }\end{array}$ & $\begin{array}{l}\text { Duration of } \\
\text { immersion } \\
\text { (hours) }\end{array}$ & $\begin{array}{l}\text { Colloid } \\
\text { concentration } \\
\text { ( } \mathbf{m g ~ m}_{\mathbf{~}}^{-1} \text { ) }\end{array}$ & $\begin{array}{l}\text { MNP content } \\
\text { of MHA } \\
\text { (wt\%) }\end{array}$ \\
\hline MHAI & 24 & 10 & 2 \\
MHA2 & 8 & 2.5 & 1 \\
MHA3 & 0.5 & 0.5 & 0.2 \\
MHA4 & 24 & 5 & 1.5 \\
MHA5 & 6 & 1 & 0.5 \\
MHA6 & 2 & 1 & 0.4 \\
\hline
\end{tabular}

Abbreviations: MHA, magnetic nanoparticle hydroxyapatite scaffold; MNP, magnetic nanoparticle.

\section{Biological testing}

Scaffold preparation for biological testing

To investigate the effect of MNP content on the cell behaviors, three types of MHA (MHA1, MHA2, and MHA3) were used for biological analysis, with the nonmagnetized samples (HA) as controls. To further investigate the cell behaviors on MHA scaffolds with the presence of exterior electromagnetic fields, cells were seeded onto MHA1 and HA, separately, and cultured under a magnetic field with an intensity of $10 \mathrm{Oe}$ and a frequency of $50 \mathrm{~Hz}$, provided by a Helmholtz coil. All the samples were prepared with weight $300 \pm 20 \mathrm{mg}$, diameter $12 \pm 0.5 \mathrm{~mm}$ and height $2 \pm 0.2 \mathrm{~mm}$. Prior to cell seeding, all the elements were continuously rinsed with PBS and cell culture medium for 24 hours. Three samples were repeated in each test group in the 3-(4,5-Dimethylthiazol-2-yl)-2, 5-diphenyltetrazolium bromide (MTT), alkaline phosphatase (ALP), and bone gla protein (BGP) assays.

\section{Cell cultivation}

Rat osteoblast cells (ROS 17/2.8) and mice preosteoblast cells (MC3T3-E1) were chosen. ROS 17/2.8 cells were cultured in complete Dulbecco's modified Eagle's medium containing $10 \% \mathrm{FBS}, 1 \%$ antibiotics (penicillin, streptomycin, amphotericin) at $37^{\circ} \mathrm{C}$ and $5 \% \mathrm{CO}_{2}$. The culture medium for MC3T3-E1 cells was alpha-modified minimum essential medium containing $10 \% \mathrm{FBS}$, and $1 \%$ antibiotics (penicillin, streptomycin, amphotericin). The samples were sterilized and put into 24-well plates. The cells were harvested at confluence with $0.25 \%$ trypsin and diluted to $10 \times 10^{4}$ cells $\mathrm{mL}^{-1}$ for scanning electron microscopy (SEM; $1 \times 10^{4}$ cells $\mathrm{mL}^{-1}$ for other tests) and then seeded onto the disks $(1 \mathrm{~mL}$ for a well) and incubated at $37^{\circ} \mathrm{C}$ with $95 \%$ air and $5 \% \mathrm{CO}_{2}$. The medium was replaced every 3 days.

\section{SEM observation}

The samples were fixed in $2.5 \%$ glutaraldehyde buffered at $\mathrm{pH}=7.4$ and dehydrated in ethanol with a series of concentrations. Samples were then critically point dried, gold sputtered, and examined with the scanning electron microscope at day 1 and day 3 .

\section{Fluorescein diacetate (FDA)/propidium} iodide (PI) staining assay

FDA and propidium iodide (PI) staining were used to assess the activity of the cells cultured on MHA and HA. At each evaluation time (1, 3, 5, and 7 days), the cell culture medium was removed and the samples were rinsed in sterile PBS. A $1 \mu \mathrm{L}$ aliquot of FDA (1 mg mL $\mathrm{mL}^{-1}$ in acetone) and $1 \mu \mathrm{L}$ 
PI (5 mg mL $\mathrm{mL}^{-1}$ in PBS) were mixed with $1 \mathrm{~mL}$ sterile PBS to stain each sample for 5 minutes. The specimens were rinsed with PBS then visualized by confocal laser scanning microscopy (CLSM).

\section{MTT assay}

The proliferation of cells was evaluated by MTT assay at days $1,3,5$, and 7. A $100 \mu \mathrm{L}$ aliquot of MTT solution ( $5 \mathrm{mg} \mathrm{mL}^{-1}$ in PBS) was added into each well and incubated for 4 hours at $37^{\circ} \mathrm{C}$ with $95 \%$ air and $5 \% \mathrm{CO}_{2}$. Then the medium was discarded and $1 \mathrm{~mL}$ dimethyl sulfoxide was added to dissolve the purple crystals. The optical density (OD) of the solution was tested with a microplate reader (Thermo Scientific Varioskan Flash; Thermo Fisher Scientific, Waltham, MA) at $570 \mathrm{~nm}$.

\section{Total protein content}

Total protein content was determined using a bicinchoninic acid reagent kit (Merck KGaA, Darmstadt, Germany) at each evaluation period $(5,7,10$, and 14 days). After discarding the medium, each well was rinsed twice with PBS. A $1 \mathrm{~mL}$ aliquot of lysis buffer was added into each well, and then frozen and thawed three times. Then $25 \mu \mathrm{L}$ of lysate was added into a 96-well plate and mixed with $200 \mu \mathrm{L}$ of work solution. After incubation at $37^{\circ} \mathrm{C}$, the $\mathrm{OD}$ value was determined at $570 \mathrm{~nm}$ and calculated according to a series of albumin standards.

\section{ALP activity}

ALP activity was determined at each time point using the p-nitrophenyl phosphate method. A $50 \mu \mathrm{L}$ aliquot of cell lysate prepared as described in the previous paragraph was mixed with $50 \mu \mathrm{L}$ p-nitrophenyl phosphate $\left(1 \mathrm{mg} \mathrm{mL}^{-1}\right)$ in the diethanolamine buffer with $\mathrm{MgCl}_{2}(\mathrm{pH}=9.8)$ and incubated at $37^{\circ} \mathrm{C}$ for 30 minutes. Then, $50 \mu \mathrm{L} \mathrm{NaOH}(2 \mathrm{M})$ was used to stop the reaction. The released p-nitrophenol was measured at $405 \mathrm{~nm}$ using the microplate reader. The values of ALP activity were normalized with respect to the total protein content.

\section{BGP activity}

BGP activity was measured using the double-antibody sandwich enzyme-linked immunosorbent assay (ELISA) method at each evaluation period. The assay was performed following the manufacturer's instructions (R\&D Systems, Minneapolis, MN).

\section{Statistical analysis}

For all experiments, analysis of variance (ANOVA) was performed using the Kruskal-Wallis ANOVA test. The $t$-test was employed to analyze the difference between the test groups in the MTT, ALP, and BGP assay results. The significance level for all samples was determined for $P<0.05$.

\section{Results and discussion Preparation of the magnetic scaffolds}

According to the method reported by Sun and Zeng, ${ }^{26}$ monodispersed MNPs surrounded with oleic acid were synthesized. The characterization results indicated that the MNPs were superparamagnetic at room temperature and the saturation magnetization $\left(\mathrm{M}_{\mathrm{s}}\right)$ of MNPs was $\sim 50 \mathrm{emu} / \mathrm{g}$. The MNPs were well dispersed in n-hexane and the average particle diameter was close to $8 \mathrm{~nm}$. The overall particle size distribution was narrow so that the particles had uniform physical and chemical properties (Figures S1-S5).

By using the microwave-assisted foaming process, HA scaffolds with interconnected macropores, and millipores on the walls of these, were fabricated. Our preliminary study showed that the diameter of the macropores ranged from 300 to $500 \mu \mathrm{m}$, the size of millipores was $1 \sim 2 \mu \mathrm{m}$, and the porosity of HA ceramics was $70 \% \sim 80 \%{ }^{25}$ This kind of HA scaffold had a specific structure with micro- and macroporosity as well as interconnectivity similar to natural bone, which is not only essential to the regeneration of tissues but also propitious for the adsorption of MNPs. ${ }^{27-30}$

The MHA were prepared by the simple immersing method. The amount of integrated MNPs was mainly influenced by the duration of immersion and the concentration of the MNP colloid. By tuning these two parameters, a series of MHAs with different MNP contents were prepared (shown in Table 1).

The integration of HA and MNPs involved immersing HA into the MNP colloid. The specific interconnected porosity of HA allowed the MNP colloid to be drawn inside the porous structure through capillary force. The subsequent drying process volatilized the hexane and kept the MNPs trapped in the structure of HA, which led to magnetization of the scaffolds while maintaining their original shape and porosity.

\section{Characterization of the magnetic scaffolds}

SEM micrographs (Figure 1) show highly porous structures for both the HA and MHA1 scaffolds. The macroscopic pores have a size of approximately several hundred microns (A and D) and appear interconnected with each other (B and E). Many fine pores (1-2 $\mu \mathrm{m}$ ) can also be observed on the walls of the macropic pores ( $\mathrm{C}$ and $\mathrm{F})$. These results indicate that both the HA and MHA scaffolds exhibit similar 

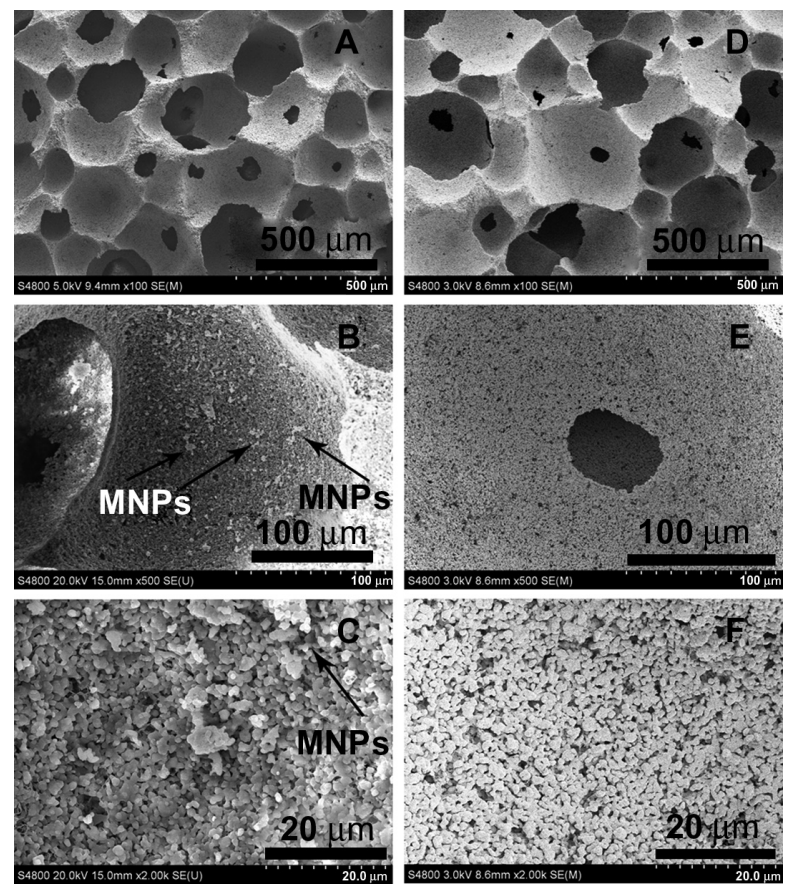

Figure I Scanning electron microscopy morphologies of MHAI (A-C) and HA (D-F) scaffolds.

Abbreviations: HA, hydroxyapatite; MHA, magnetic nanoparticle hydroxyapatite scaffold; MNP, magnetic nanoparticle.

macro- and microporosity and good interconnectivity. No evident change in the porosity characteristics was identified, suggesting little change as a result of the immersing process. The scaffold structure also mimicked the morphology of natural spongy bone. ${ }^{31}$ White spots are clearly visible in the SEM micrographs (B and C) and are likely to be representative of iron oxide. Energy-dispersive spectroscopy spectra (Figure S6) confirmed the presence of iron as well as calcium and phosphorus in MHA1. The $\mathrm{Ca} / \mathrm{P}$ ratios of both MHA1 (1.680) and HA (1.673) were very close to the theoretical value of 1.67 .

XRD patterns (Figure 2) show that both the MHA1 and HA scaffolds had the same crystal structure and the peak positions matched well with those of the standard HA, indicating that the process did not lead to the formation of any secondary crystalline phase or change in the HA structure. However, due to the low MNP content ( $2 \mathrm{wt} \%$ ) in MHA and their nanoscale, no magnetite nanocrystal peak is observed in the XRD spectra.

The hysteresis loops of the MHA with three different MNP contents are shown in Figure 3. All the curves passed through the zero point and showed no coercivity or remanence, typical of superparamagnetic behavior, indicating that the presence of MNPs in HA led to the superparamagnetism of the scaffolds. ${ }^{25}$ The $\mathrm{M}_{\mathrm{s}}$ of these three types of MHA were

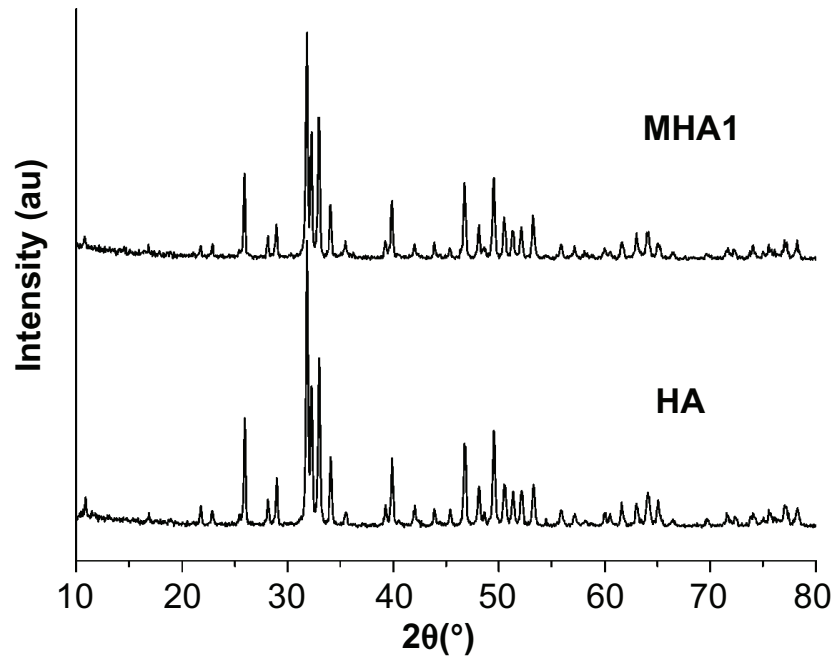

Figure $2 \mathrm{X}$-ray diffraction patterns of MHAI and HA scaffolds. Abbreviations: MHA, magnetic hydroxyapatite scaffolds; HA, hydroxyapatite.

$0.94,0.53$, and $0.24 \mathrm{emu} / \mathrm{g}$, respectively, which agrees with the calculated values according to the saturation magnetization of the MNPs $(50 \mathrm{emu} / \mathrm{g}$ ) and the respective MNP contents of the MHA scaffolds. Therefore, the magnetic properties of the MHA depended primarily on the MNP content in the scaffold.

\section{Stability of MHA under soaking in different biological solutions}

The quantity of MNPs released from MHA1 was analyzed by atomic absorption spectroscopy by measuring the iron content of the retrieved soaked solution. The calculated masses of the released magnetite from the MHA soaking in $\mathrm{H}_{2} \mathrm{O}$, PBS, SBF, and FBS are shown as a function of time in Figure 4. These curves show a rapid release in the first 10 hours then a more

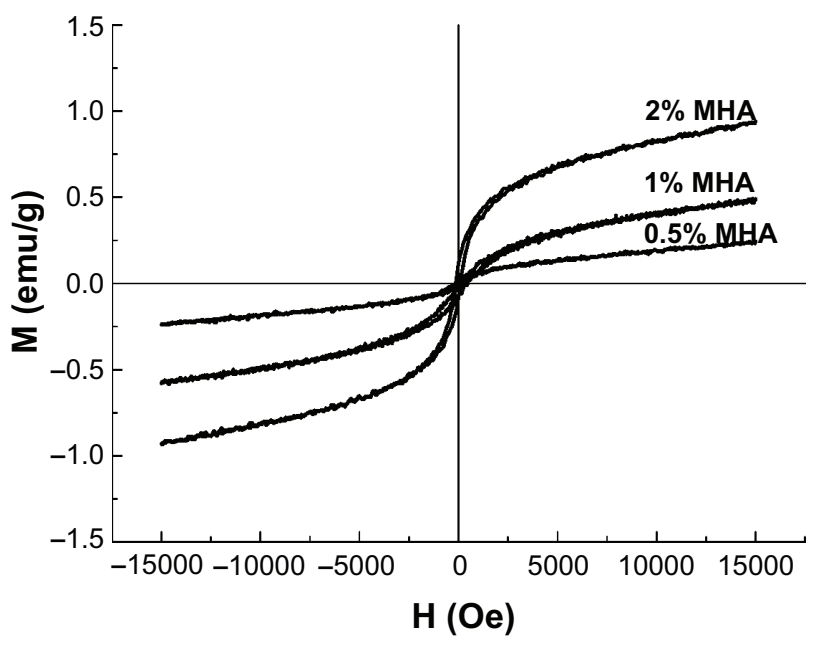

Figure 3 Magnetization curves of magnetic nanoparticle hydroxyapatite scaffolds (MHAs) with three different magnetic nanoparticle contents $(2 \%, 1 \%$, and $0.5 \%)$. 

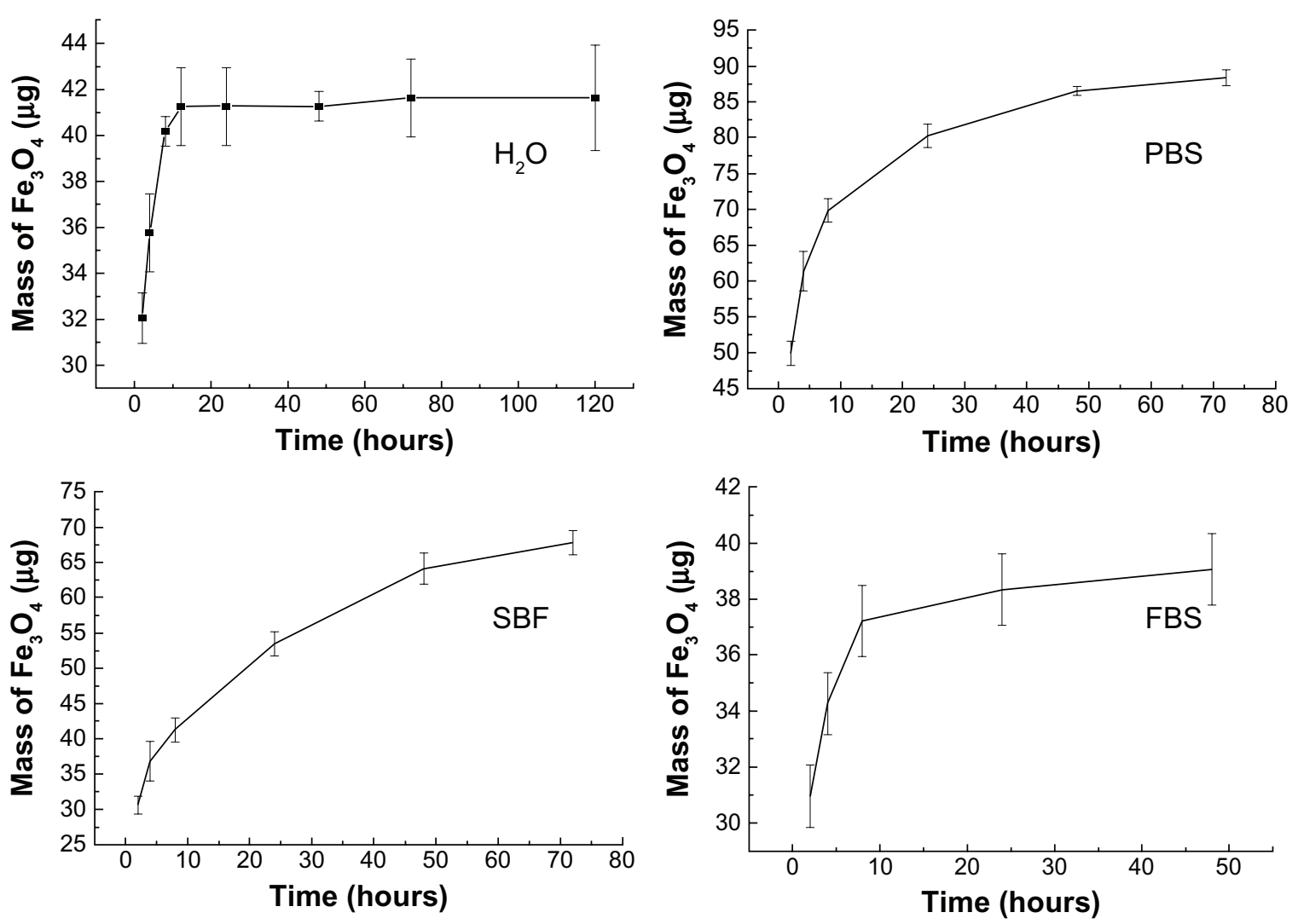

Figure 4 Released magnetite mass-time curves of MHAI soaking in $\mathrm{H}_{2} \mathrm{O}, \mathrm{PBS}, \mathrm{SBF}$, and FBS separately.

Abbreviations: MHA, magnetic nanoparticle hydroxyapatite scaffold; PBS, phosphate-buffered saline; SBF, simulated body fluids; FBS, fetal bovine serum.

gradual release over a longer period. Finally, MHA1 released $2.1 \%, 4.3 \%, 3.4 \%$, and $2.0 \%$ of the adsorbed magnetite in $\mathrm{H}_{2} \mathrm{O}, \mathrm{PBS}, \mathrm{SBF}$, and FBS, respectively. The initial rapid release in SBF was not as obvious when compared with the other three types of media, which may be attributed to the higher concentration of inorganic salts in SBF.

The integration of HA and MNPs involved an adsorptiondesorption dynamic equilibrium process. The observed rapid increase of released magnetite in the first 10 hours was probably due to a quick desorption process upon contact with biological media. However, the release curves flattened out at an extremely low percentage $(<5 \%)$ of released magnetite, which suggests that the specific porous structure and the large surface energy of HA helped to retain the MNPs in the scaffold, leading to the inhibition of the desorption process. These results confirm that MHA would be a good candidate for in vivo applications, since the nanoparticle contents within the scaffolds remained almost constant under physiological conditions.

\section{Biological testing} SEM

SEM pictures (Figure 5) show the adhesion of MC3T3-E1 and ROS 17/2.8 cells on HA and MHA scaffolds. Both the
ROS 17/2.8 and MC3T3-E1 cells spread well and attached firmly on the surfaces of HA and MHA scaffolds, indicating good biocompatibility for both scaffolds. The cells also appeared to penetrate inside the pore structure during the first day of cultivation. The cell number at 3 days was significantly greater than that at 1 day for each type of scaffold and cell, confirming the activity of cell proliferation processes on the surface and into the pores of all scaffolds. The number of cells on MHA1 was larger than that on HA at each time point, and cells on MHA1 were better spread than those on HA. The quality of cell adhesion is known to greatly influence the proliferation and differentiation of cells. ${ }^{32}$ Thus, the results indicated that the MHA scaffold has superior biocompatibility compared with the HA scaffold.

\section{FDA/PI staining assay}

The viability and proliferation of MC3T3-E1 and ROS $17 / 2.8$ cells cultured on the magnetic scaffolds are demonstrated in the CLSM images after FDA/PI staining (Figures 6 and 7). In the CLSM images, the red spots were scored as unviable cells and the green as viable cells. Almost no red spots were observed in the CLSM images, indicating that both the MHA1 and HA scaffolds caused low levels 


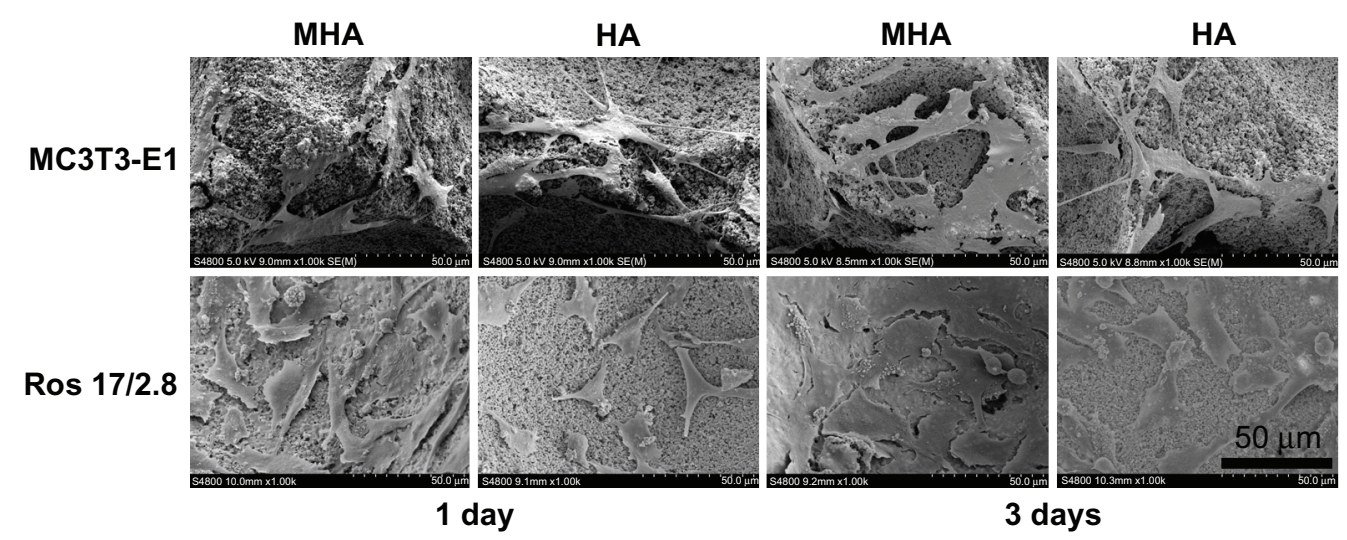

Figure 5 Scanning electron microscopy micrographs illustrating MC3T3-EI and ROS I7/2.8 cell growth on MHAI and HA after I and 3 days of cultivation. Abbreviations: MHA, magnetic nanoparticle hydroxyapatite scaffold; HA, hydroxyapatite.

of nonviable cells. This result confirms the excellent biocompatibility of both MHA1 and HA. The layer-by-layer scanning analysis (data not shown) suggests that there were a considerable number of green spots around the micropores on each layer, which indicates that the cells first attached to the surface of the scaffolds then grew along the wall of the micropores and extended into the porous structure.

Figure 6 shows that the number of viable cells increased with culture time in all four test groups (columns A-D). Interestingly, the ROS 17/2.8 cells proliferated well after

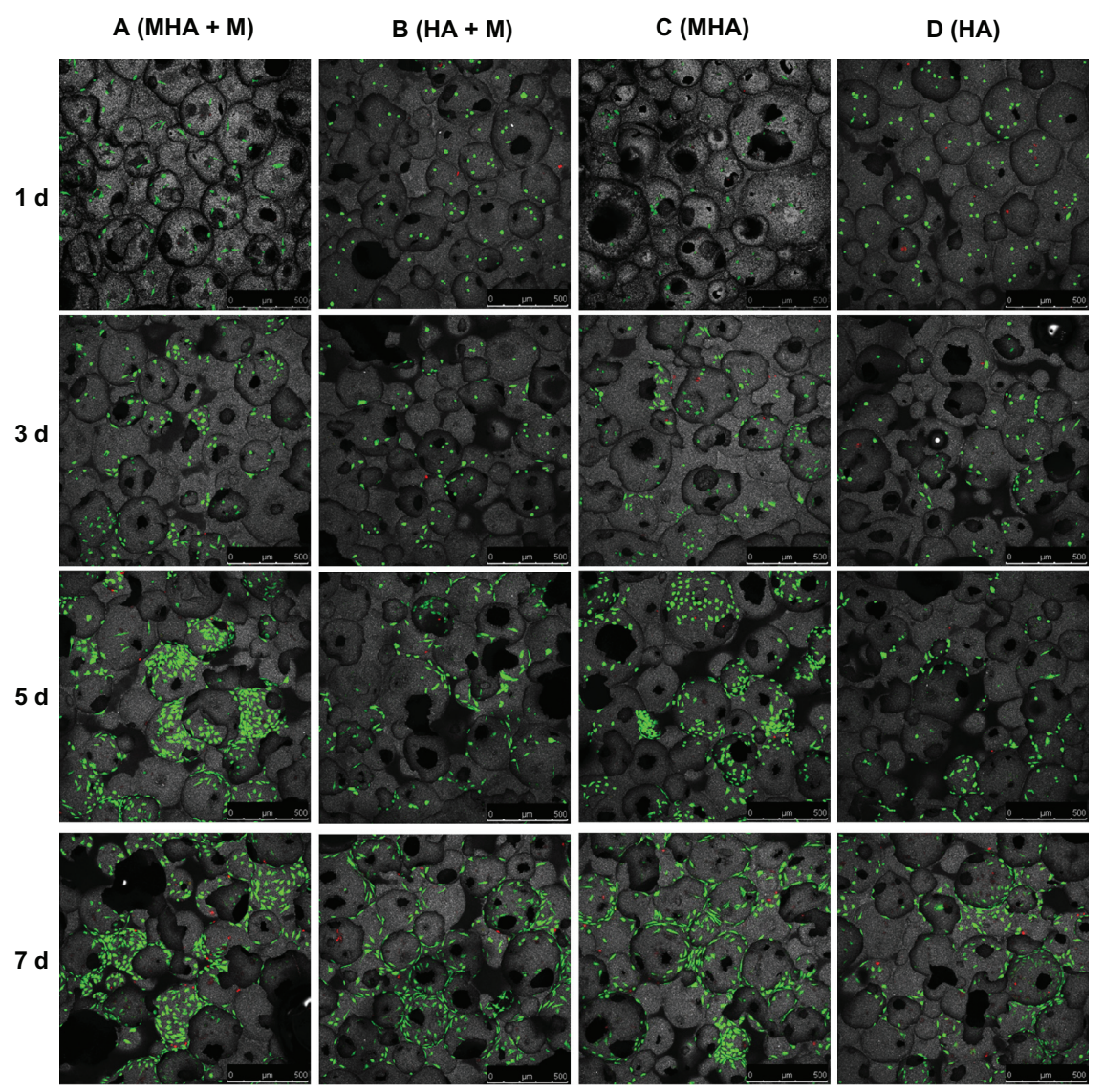

Figure 6 Confocal laser scanning microscopy images of ROS 17/2.8 cells stained with fluorescein diacetate/propidium iodide after cultivation on the MHAI (columns A and C) and HA (columns $\mathbf{B}$ and $\mathbf{D}$ ) for I, 3, 5, and 7 days.

Notes: Half of the samples were cultured under the exterior magnetic field (columns $\mathbf{A}$ and $\mathbf{B}$ ), denoted as MHAI + M and HA + M, and the other half were cultured without the exterior magnetic field (columns $\mathbf{C}$ and $\mathbf{D}$ ).

Abbreviations: MHA, magnetic nanoparticle hydroxyapatite scaffold; HA, hydroxyapatite scaffold. 


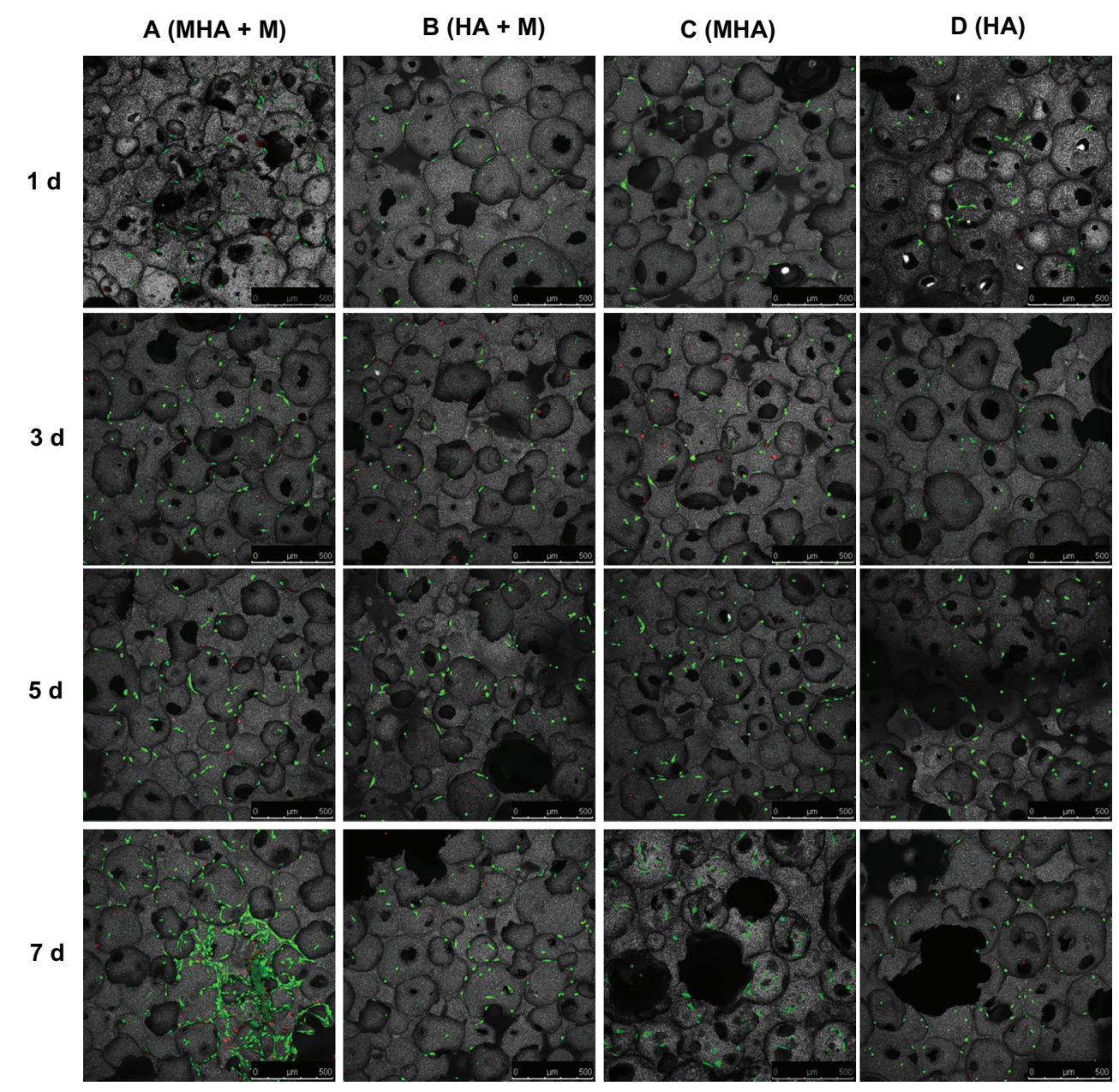

Figure 7 Confocal laser scanning microscopy images of MC3T3-EI cells stained with fluorescein diacetate/propidium iodide after cultivation on MHAI (columns $\mathbf{A}$ and $\mathbf{C}$ ) and HA (columns B and D) for I, 3, 5, and 7 days.

Notes: Half of the samples were cultured under the exterior magnetic field (columns $\mathbf{A}$ and $\mathbf{B}$ ), denoted as MHAI + M and HA + M, and the other half were cultured without the exterior magnetic field (columns $\mathbf{C}$ and $\mathbf{D}$ ).

Abbreviations: MHA, magnetic nanoparticle hydroxyapatite scaffold; HA, hydroxyapatite scaffold.

being seeded on MHA and HA scaffolds. Furthermore, at each time point, the number of viable cells in the four test groups followed the rule $\mathrm{A}>\mathrm{C}>\mathrm{B}>\mathrm{D}$. Under each culture condition (with or without exterior magnetic fields), the number of viable cells on the MHA scaffold was higher than that on the HA scaffold (A $>$ B and C $>$ D), suggesting enhanced cell proliferation on the MHA scaffold due to the incorporation of MNPs. For each type of scaffold (MHA or HA), the number of viable cells under the exterior magnetic fields was higher than that without the exterior magnetic fields ( $\mathrm{A}>\mathrm{C}$ and $\mathrm{B}>\mathrm{D}$ ), which indicates that the exterior magnetic field improved the cell proliferation on both the MHA and HA scaffolds, consistent with previous research. ${ }^{2,33,34}$ The most significant cell proliferation was found on the MHA scaffold with the exterior magnetic fields, suggesting a likely synergistic effect between the MHA and the exterior magnetic fields. A similar trend is observed for the MC3T3-E1 cells in Figure 7. However, the trend is less significant than that in Figure 6, which is probably due to the overall lower proliferation rate of the MC3T3-E1 cells compared with that of the ROS 17/2.8 cells.

\section{MTT assay}

MTT assay was used to evaluate the proliferation of ROS 17/2.8 and MC3T3-E1 cells. As already explained, to investigate the influence of the MNP content of MHA on cell proliferation, three types of MHA scaffolds (MHA1, MHA2, and MHA3) with different MNP contents (2\%, $1 \%, 0.2 \%$ ) were used in the study, with nonmagnetized samples used (HA) as a control. The OD values increased with time in all test groups (Figure 8), confirming the excellent biocompatibility of the MHA and HA scaffolds. This result is consistent with the analysis of the SEM and CLSM observations. However, both cell types proliferated on the 
A

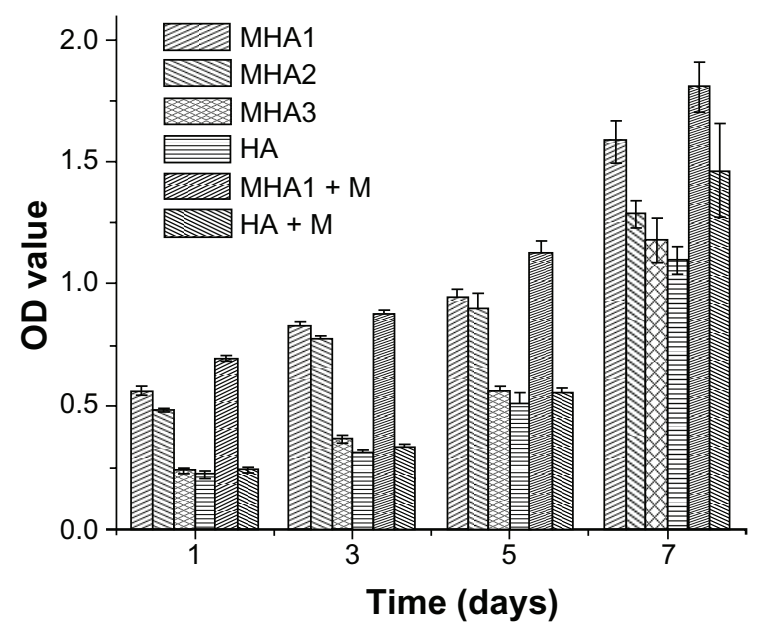

B

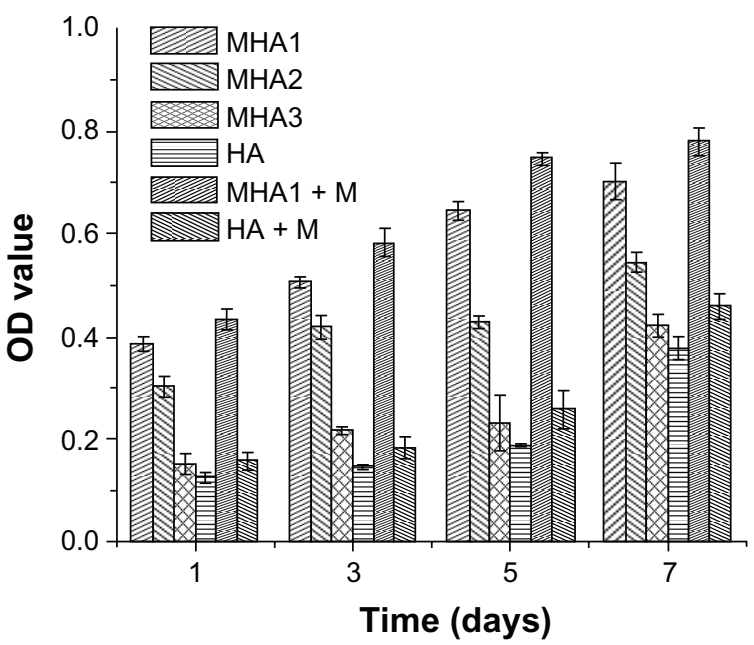

Figure 8 MTT assay results of cells, (A) ROS I7/I.8 and (B) MC3T3-EI, after cultivation with all types of scaffolds for I, 3, 5, and 7 days.

Notes: The cells were also cultured on MHAI and HA under exterior magnetic fields, denoted as MHAI $+\mathrm{M}$ and $\mathrm{HA}+\mathrm{M}$, respectively. The mean values were calculated from the average results of three samples, the error bars represent \pm standard deviation.

Abbreviations: MTT, 3-(4,5-Dimethylthiazol-2-yl)-2,5-diphenyltetrazolium bromide; MHA, magnetic nanoparticle hydroxyapatite scaffold; HA, hydroxyapatite scaffold; OD, optical density.

MHA scaffolds (from MHA1 to MHA3) had higher OD values than those on HA at the same culture time. The difference of $\mathrm{OD}$ values between the magnetic and ordinary scaffold groups was significant $(P<0.05)$ except for some of those between MHA3 and HA, which was probably due to the low MNP content of MHA3. The maximum increase of OD value reached $179 \%$ (MC3T3-E1 co-cultivated on MHA1 for 5 days). Furthermore, the OD value at each time point increased with the MNP content of MHA for both cell types (MHA1 > MHA2 > MHA3), suggesting a positive correlation between the magnetite content and the cell proliferation. It was suggested that each magnetic nanoparticle could be considered as a single magnetic domain in a nanoscale, which might affect the ion channels on the cell membrane so as to influence the cell behaviors. ${ }^{35-37}$ Despite the intensity of the nano-sized magnetic field being extremely low, the total effect would likely be strengthened with an increase of the amount of MNPs, thus it would have a stronger influence on cell response.

The cells cultured on the MHA1 and HA scaffolds under the exterior magnetic fields had OD values higher than those under the normal culture condition. A significant difference $(P<0.05)$ of the OD values can be found between the MHA1 under the exterior magnetic field and MHA1 samples at all culture times for both cell types. In contrast, some of the differences in OD values between the HA under the exterior magnetic field and HA samples were not significant. It suggests that the exterior magnetic field had a beneficial effect on stimulating the cell proliferation, but the enhancement was limited in HA samples due to the low intensity of the exterior magnetic fields, while this stimulating effect was magnified in the presence of MNPs. Consistent with the CLSM image result, this result again suggests a synergistic effect between the MHA scaffold and the exterior magnetic field.

\section{ALP and BGP activity assay}

ALP and BGP tests were used to assess the osteoblast phenotype expression of ROS 17/2.8 and MC3T3-E1 cells. As shown in Figure 9, for ROS 17/2.8 cell line, no statistically significant differences in ALP activities $(P>0.05)$ were observed among all test groups at 5, 7, and 10 days. Significant differences $(P<0.05)$ were only observed between the MHA and HA scaffolds at 14 days. The ALP value of MHA was higher than that of HA by $16.3 \%$ under ordinary culture conditions and by $31.5 \%$ under the exterior magnetic field. For the MC3T3-E1 cell line, the ALP values of cells cultured with MHA were significantly higher than those cultured on HA at 5, 7, and 10 days, and most of the samples cultured under the exterior magnetic field had significantly higher ALP values than those under ordinary culture conditions at each time point.

The result of the ALP assay indicates that the MHA scaffold improved the osteoblast phenotype expression of ROS $17 / 2.8$ cells at the final period of the cultivation more than the HA scaffold, and the improvement was further enhanced by the exterior magnetic field. Both the MHA and the exterior magnetic field improved the osteogenic differentiation of MC3T3-E1 cells from the beginning of the cultivation. 

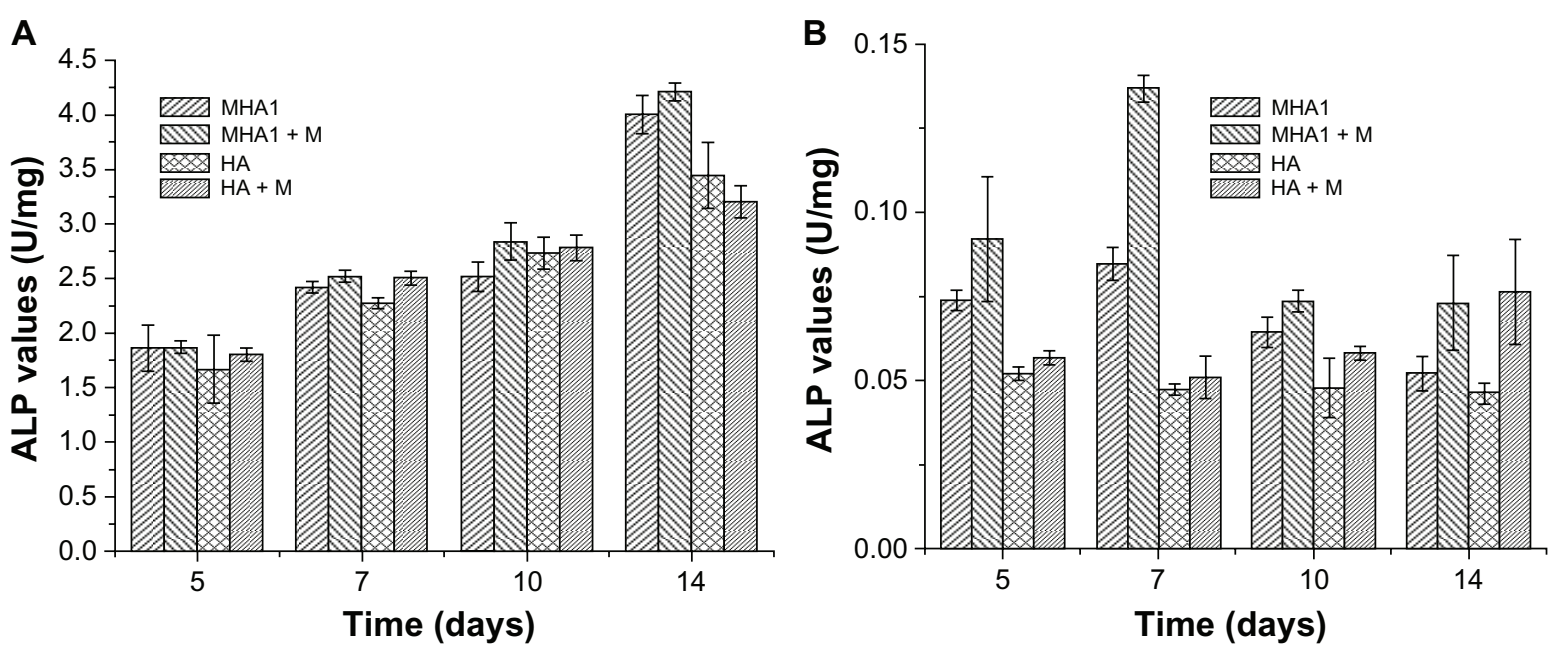

Figure 9 Alkaline phosphatase (ALP) activity test results of cells, (A) ROS I7/I.8 and (B) MC3T3-EI, after cultivation with MHAI and HA for 5, 7, I0, and I4 days. Notes: The samples were divided into four test groups: HA and MHA with and without exterior magnetic fields, respectively. "+M" stands for cultivation under exterior magnetic fields. The mean values were calculated from the average results of three samples, the error bars represent \pm standard deviation.

Abbreviations: ALP, alkaline phosphatase; MHA, magnetic nanoparticle hydroxyapatite scaffold; HA, hydroxyapatite scaffold.

This can be attributed to the difference between the two cell types, as ROS 17/2.8 is an osteoblast cell while MC3T3-E1 is a preosteoblast cell.

As shown in Figure 10, there were no statistically significant differences in BGP values of the MC3T3-E1 cells among all testing groups at any time points. For the ROS 17/2.8 cell line, there were also no statistically significant differences in BGP values among all test groups at 5 and 7 days. After 10 days of cultivation, both the MHA and HA samples cultured under the exterior magnetic field had significantly higher BGP values than those under ordinary culture conditions. Furthermore, under exterior magnetic fields, the BGP value of MHA was significantly higher than that of HA, but the difference between the MHA and HA scaffolds under the ordinary culture conditions was not significant. After 14 days of cultivation, these differences became more obvious; the differences between the MHA and HA scaffolds under both the ordinary culture conditions and the exterior magnetic field became significant.

The result of the BGP assay indicates that both the exterior magnetic field and the MHA possessed some stimulating effect on the osteoblast phenotype expression of the bone cells. There may be some synergistic effect between the MHA and the exterior magnetic field. The result further confirms that the magnetic scaffolds responded to the exterior magnetic field to intensify the stimulating effect.

By adding MNPs into the HA scaffold, the magnetic therapy function was introduced into the material.
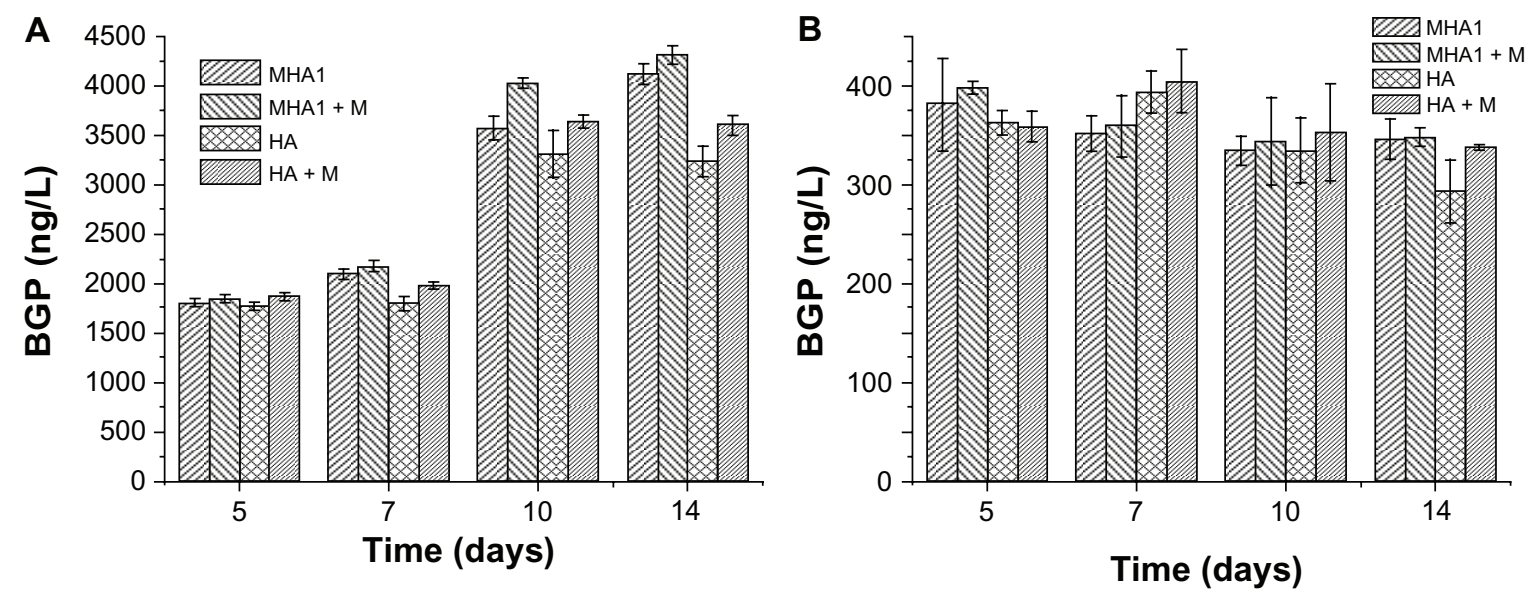

Figure 10 Bone gla protein (BGP) activity test results of cells, (A) ROS I7/I.8 and (B) MC3T3-EI, after cultivation with MHAI and HA for 5, 7, I0, and I4 days. Notes: The samples were divided into four test groups: HA and MHA with and without exterior magnetic field, respectively. The sign $+M$ stands for cultivation under exterior magnetic fields. The mean values were calculated from the average results of three samples, the error bars represent \pm standard deviation.

Abbreviations: MHA, magnetic nanoparticle hydroxyapatite scaffold; HA, hydroxyapatite scaffold. 
The magnetic scaffold had some beneficial effect on stimulating cell adhesion, proliferation, and differentiation. This effect was intensified with the increase in MNP content, suggesting that the magnetic scaffold has great potential to achieve intrinsic magnetic therapy. Furthermore, the magnetic scaffold might respond to external magnetic fields and demonstrate some synergistic effect to further improve the cell response, indicating the potential for enhanced magnetic therapy. Nevertheless, many issues are still unclear, such as the interaction between MNPs and cells, the mechanism of how the exterior magnetic field influences cell activities, and the synergistic effect between the exterior magnetic field and MHA. Moreover, in vivo experiments are needed to completely demonstrate the biocompatibility and osteoinductivity of the MHA scaffolds (in progress).

\section{Conclusion}

This study introduced the MHA scaffold as a type of bone graft substitute. MHA scaffolds with various MNP contents were fabricated by tuning the duration of immersion and the colloid concentration. The physical and chemical properties of MHA and its excellent stability under physiological conditions indicate its potential as an alternative bone graft substitute.

The capability of these new magnetic scaffolds to improve bone formation and growth was assayed. Our in vitro results demonstrate that the magnetic HA scaffold stimulated cell adhesion, proliferation, and differentiation by itself, which can be attributed to the intrinsic nanoscale magnetic field provided by the incorporated MNPs. A positive correlation of the cell response with the MNP content was observed in the MHA scaffolds. The result also indicates that the exterior magnetic field stimulated the proliferation and differentiation of cells and the MHA responded with the exterior magnetic field and exhibited further enhanced cell proliferation and differentiation, probably due to the synergistic effect between the incorporated MNPs and the exterior magnetic field.

This new type of scaffold certainly has the potential to be considered as a bone graft substitute with an attractive new property: magnetic therapy, providing a better alternative to conventional scaffolds.

\section{Acknowledgments}

The authors would like to thank National Basic Research Program of China (National 973 program, Nos 2011CB606206, 2012CB619103), the National Natural Science Foundation of China (31070849, 51133004), the Department of Science and Technology of Sichuan Province (2009HH0001, 2009SZ0137), and the Ministry of Science and Technology (2010DFA51550).

\section{Disclosure}

The authors report no conflicts of interest in this work.

\section{References}

1. Bassett CA, Schink-Ascani M, Lewis SM. Effects of pulsed electromagnetic fields on Steinberg ratings of femoral head osteonecrosis. Clin Orthop Relat Res. 1989;(246):172-185.

2. Santini MT, Rainaldi G, Ferrante A, Indovina PL, Vecchia P, Donelli G. Effects of a $50 \mathrm{~Hz}$ sinusoidal magnetic field on cell adhesion molecule expression in two human osteosarcoma cell lines (MG-63 and Saos-2). Bioelectromagnetics. 2003;24(5):327-338.

3. McLeod KJ, Collazo L. Suppression of a differentiation response in MC-3T3-E1 osteoblast-like cells by sustained, low-level, $30 \mathrm{~Hz}$ magnetic-field exposure. Radiat Res. 2000;153(5 Pt 2):706-714.

4. Jansen JH, van der Jagt OP, Punt BJ, et al. Stimulation of osteogenic differentiation in human osteoprogenitor cells by pulsed electromagnetic fields: an in vitro study. BMC Musculoskelet Disord. 2010;11:188.

5. Fini M, Cadossi R, Canè V, et al. The effect of pulsed electromagnetic fields on the osteointegration of hydroxyapatite implants in cancellous bone: a morphologic and microstructural in vivo study. J Orthop Res. 2002;20(4):756-763.

6. Zhang XY, Xue Y, Zhang Y. Effects of $0.4 \mathrm{~T}$ rotating magnetic field exposure on density, strength, calcium and metabolism of rat thigh bones. Bioelectromagnetics. 2006;27(1):1-9.

7. Chang K, Chang WH. Pulsed electromagnetic fields prevent osteoporosis in an ovariectomized female rat model: a prostaglandin E2-associated process. Bioelectromagnetics. 2003;24(3):189-198.

8. Taylor KF, Inoue N, Rafiee B, Tis JE, McHale KA, Chao EY. Effect of pulsed electromagnetic fields on maturation of regenerate bone in a rabbit limb lengthening model. J Orthop Res. 2006;24(1):2-10.

9. Sun S, Zeng H, Robinson DB, et al. Monodisperse $\mathrm{MFe}_{2} \mathrm{O}_{4}(\mathrm{M}=\mathrm{Fe}$, Co, Mn) nanoparticles. J Am Chem Soc. 2004;126(1):273-279.

10. Wang L, Yang Z, Gao J, et al. A biocompatible method of decorporation: bisphosphonate-modified magnetite nanoparticles to remove uranyl ions from blood. J Am Chem Soc. 2006;128(41):13358-13359.

11. Kim J, Lee JE, Lee J, et al. Magnetic fluorescent delivery vehicle using uniform mesoporous silica spheres embedded with monodisperse magnetic and semiconductor nanocrystals. J Am Chem Soc. 2005;128(3): 688-689.

12. Jun YW, Huh YM, Choi JS, et al. Nanoscale size effect of magnetic nanocrystals and their utilization for cancer diagnosis via magnetic resonance imaging. J Am Chem Soc. 2005;127(16):5732-5733.

13. Bretcanu O, Verné E, Cöisson M, Tiberto P, Allia P. Magnetic properties of the ferrimagnetic glass-ceramics for hyperthermia. J Magn Magn Mater. 2006;305(2):529-533.

14. Wei Y, Zhang X, Song Y, et al. Magnetic biodegradable Fe3O4/CS/ PVA nanofibrous membranes for bone regeneration. Biomed Mater. 2011;6(5):055008.

15. Meng J, Zhang Y, Qi X, et al. Paramagnetic nanofibrous composite films enhance the osteogenic responses of pre-osteoblast cells. Nanoscale. 2010;2(12):2565-2569.

16. Bañobre-López M, Piñeiro-Redondo Y, De Santis R, et al. Poly(caprolactone) based magnetic scaffolds for bone tissue engineering. J Appl Phys. 2011;109(7):07B313.

17. Wu C, Fan W, Zhu Y, et al. Multifunctional magnetic mesoporous bioactive glass scaffolds with a hierarchical pore structure. Acta Biomater. 2011;7(10):3563-3572.

18. Tampieri A, Landi E, Valentini F, et al. A conceptually new type of bio-hybrid scaffold for bone regeneration. Nanotechnology. 2011; 22(1):015104. 
19. Tampieri A, D'Alessandro T, Sandri M, et al. Intrinsic magnetism and hyperthermia in bioactive Fe-doped hydroxyapatite. Acta Biomater. 2012;8(2):843-851.

20. De Santis R, Gloria A, Russo T, et al. A basic approach toward the development of nanocomposite magnetic scaffolds for advanced bone tissue engineering. J Appl Polym Sci. 2011;122(6):3599-3605.

21. Bock N, Riminucci A, Dionigi C, et al. A novel route in bone tissue engineering: magnetic biomimetic scaffolds. Acta Biomater. 2010;6(3): 786-796.

22. Jun YW, Seo JW, Cheon J. Nanoscaling laws of magnetic nanoparticles and their applicabilities in biomedical sciences. Acc Chem Res. 2008;41(2):179-189.

23. Jeong U, Teng X, Wang Y, Yang H, Xia Y. Superparamagnetic colloids: controlled synthesis and niche applications. Adv Mater. 2007;19(1): 33-60.

24. Gupta AK, Gupta M. Synthesis and surface engineering of iron oxide nanoparticles for biomedical applications. Biomaterials. 2005;26(18): 3995-4021.

25. Wu Y, Jiang W, Wen X, et al. A novel calcium phosphate ceramicmagnetic nanoparticle composite as a potential bone substitute. Biomed Mater. 2010;5(1):15001.

26. Sun S, Zeng H. Size-controlled synthesis of magnetite nanoparticles. J Am Chem Soc. 2002;124(28):8204-8205.

27. Schieker M, Seitz H, Drosse I, Seitz S, Mutschler W. Biomaterials as scaffold for bone tissue engineering. European Journal of Trauma. 2006;32(2):114-124.

28. Hing KA. Bioceramic bone graft substitutes: influence of porosity and chemistry. International Journal of Applied Ceramic Technology. 2005;2(3):184-199.
29. Vallet-Regí M. Ceramics for medical applications. Journal of the Chemical Society, Dalton Transactions. 2001(2):97-108.

30. Vallet-Regí M. Revisiting ceramics for medical applications. Dalton Trans. 2006;(44):5211-5220.

31. Yuan H, van Blitterswijk CA, de Groot K, de Bruijn JD. Cross-species comparison of ectopic bone formation in biphasic calcium phosphate (BCP) and hydroxyapatite (HA) scaffolds. Tissue Eng. 2006;12(6): 1607-1615.

32. Rodrigues CV, Serricella P, Linhares AB, et al. Characterization of a bovine collagen-hydroxyapatite composite scaffold for bone tissue engineering. Biomaterials. 2003;24(27):4987-4997.

33. De Mattei M, Caruso A, Traina GC, Pezzetti F, Baroni T, Sollazzo V. Correlation between pulsed electromagnetic fields exposure time and cell proliferation increase in human osteosarcoma cell lines and human normal osteoblast cells in vitro. Bioelectromagnetics. 1999;20(3): 177-182.

34. Chang WH, Chen LT, Sun JS, Lin FH. Effect of pulse-burst electromagnetic field stimulation on osteoblast cell activities. Bioelectromagnetics. 2004;25(6):457-465.

35. Hughes S, EI Haj AJ, Dobson J. Magnetic micro- and nanoparticle mediated activation of mechanosensitive ion channels. Med Eng Phys. 2005;27(9):754-762.

36. Huang DM, Hsiao JK, Chen YC, et al. The promotion of human mesenchymal stem cell proliferation by superparamagnetic iron oxide nanoparticles. Biomaterials. Aug 2009;30(22):3645-3651.

37. Shen JF, Chao YL, Du L. Effects of static magnetic fields on the voltagegated potassium channel currents in trigeminal root ganglion neurons. Neurosci Lett. March 26, 2007;415(2):164-168. 


\section{Supplementary figures}

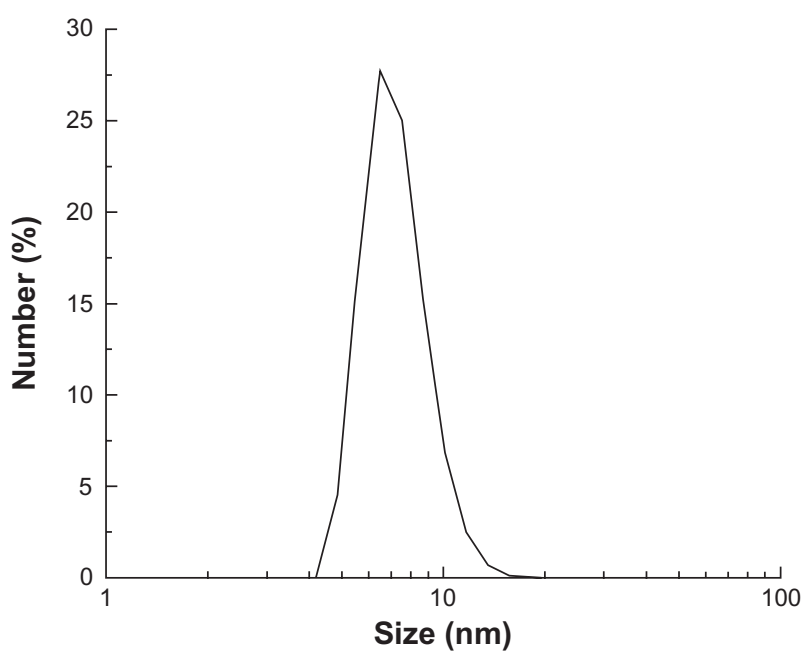

Figure SI Particle size of MNPs.

Abbreviation: MNPs, magnetic nanoparticles.

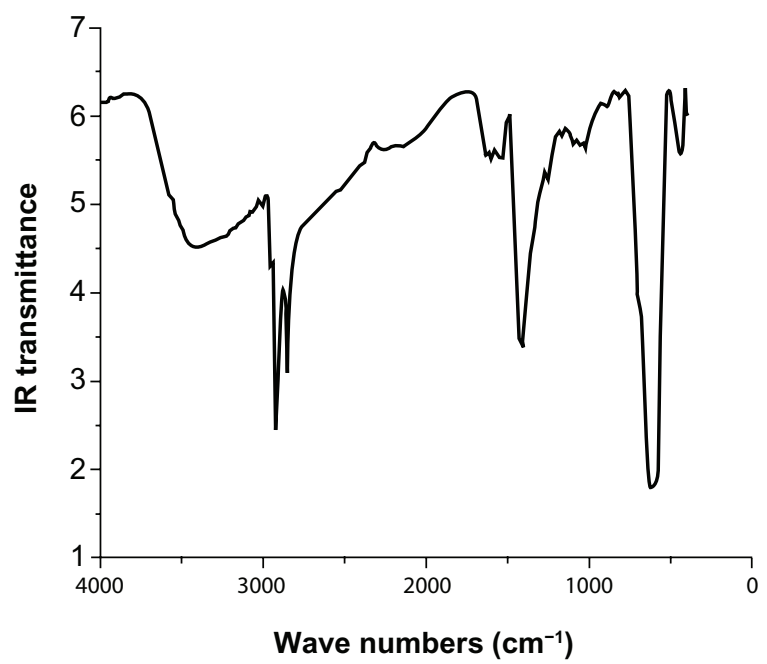

Figure S2 FTIR spectra of MNPs.

Abbreviation: IR, infrared; FTIR, fourier transform infrared; MNP, magnetic nanoparticles.

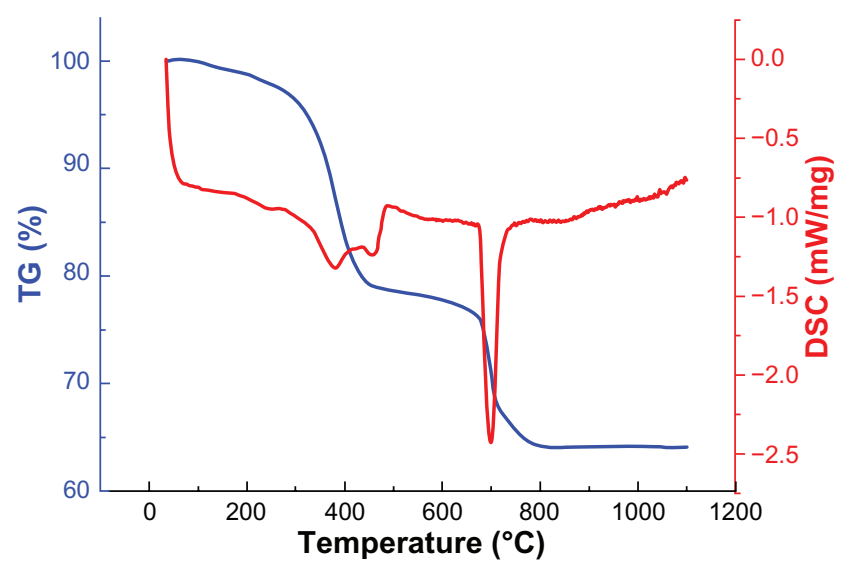

Figure S3 TG and DSC curves of MNPs.

Abbreviations: DSC, differential scanning calorimetry; TG, thermo gravimetric; MNPs, magnetic nanoparticles.

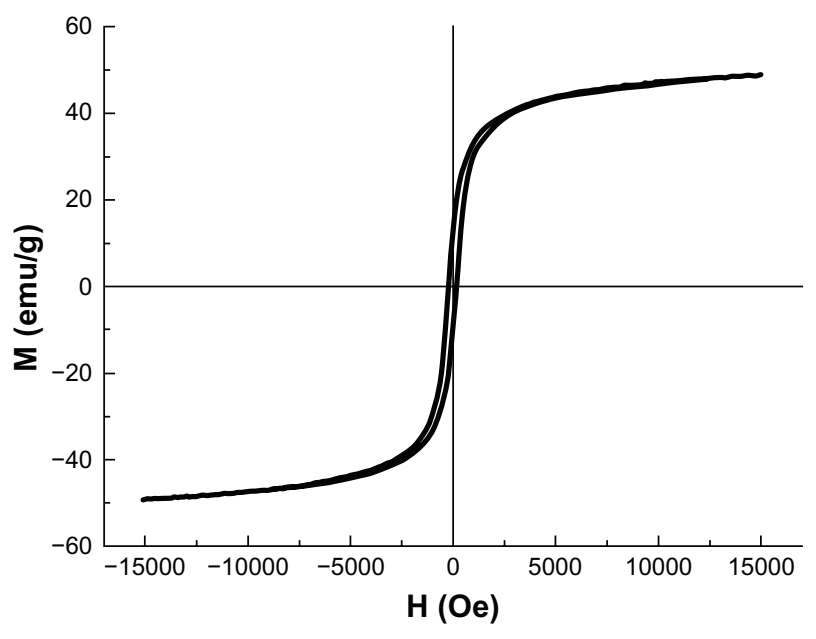

Figure S4 Magnetization curves of MNPs.

Abbreviation: MNPs, magnetic nanoparticles.

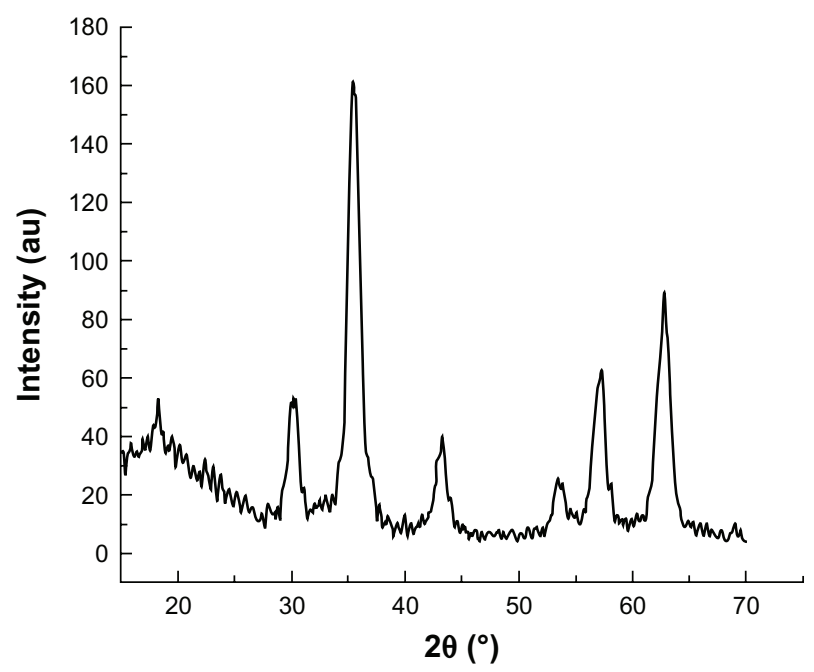

Figure S5 XRD pattern of MNPs.

Abbreviations: XRD, X-ray diffraction; MNPs, magnetic nanoparticles. 


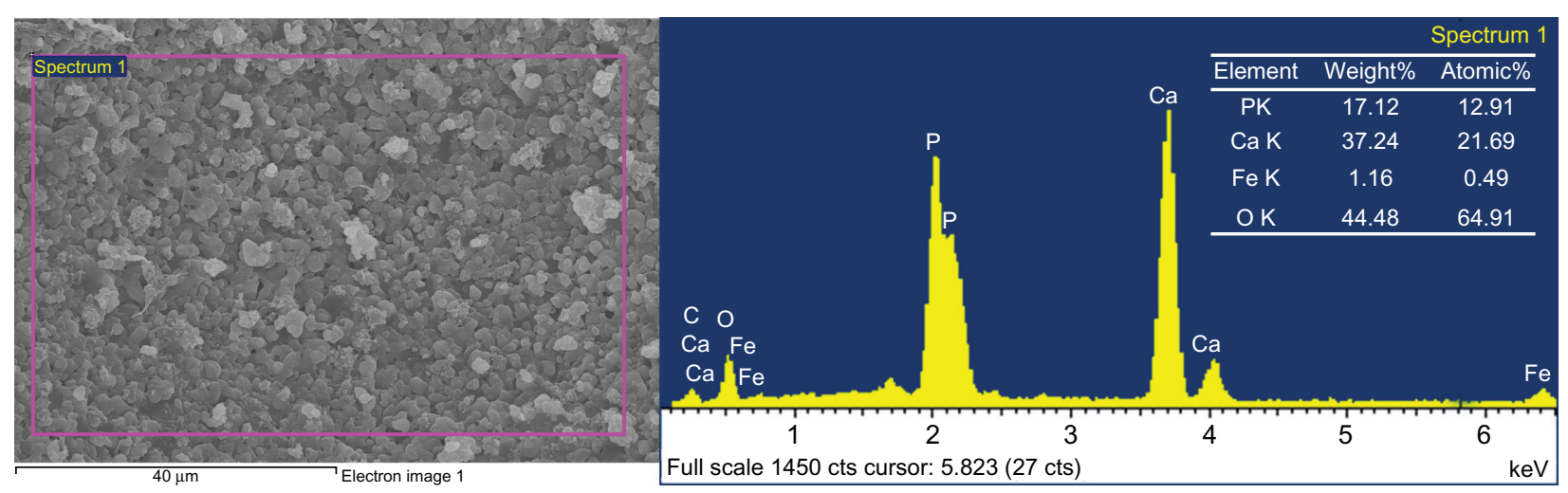

Figure S6 SEM image (left) and EDS spectra (right) of MHAI scaffold.

Abbreviations: SEM, scanning electron microscope; EDS, energy dispersive spectrometer; MHA, magnetic hydroxyapatite.
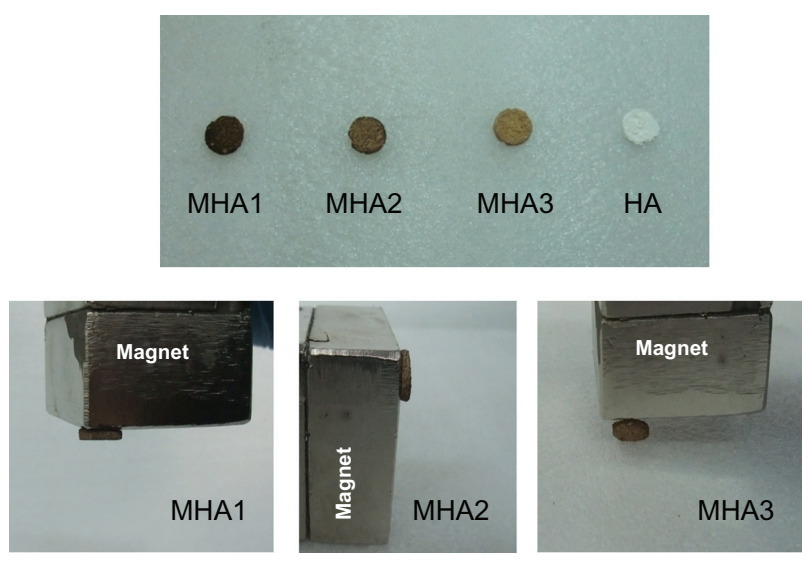

Figure S7 Picture of the magnetic scaffolds to illustrate the magnetic property of MHA on a macroscale.

Abbreviation: MHA, magnetic hydroxyapatite.

\section{Publish your work in this journal}

The International Journal of Nanomedicine is an international, peerreviewed journal focusing on the application of nanotechnology in diagnostics, therapeutics, and drug delivery systems throughout the biomedical field. This journal is indexed on PubMed Central, MedLine, CAS, SciSearch ${ }^{\circledR}$, Current Contents ${ }^{\circledR} /$ Clinical Medicine,
Journal Citation Reports/Science Edition, EMBase, Scopus and the Elsevier Bibliographic databases. The manuscript management system is completely online and includes a very quick and fair peer-review system, which is all easy to use. Visit http://www.dovepress.com/ testimonials.php to read real quotes from published authors.

Submit your manuscript here: http://www.dovepress.com/international-journal-of-nanomedicine-journal 